\title{
Prevalence and factors associated with suboptimal peak inspiratory flow rates in COPD
}

This article was published in the following Dove Medical Press journal: International Journal of COPD

\author{
Sohini Ghosh' \\ Roy A Pleasants ${ }^{2}$ \\ Jill A Ohar \\ James F Donohue' \\ M Bradley Drummond' \\ 'Division of Pulmonary Diseases \\ and Critical Medicine, Department \\ of Medicine, University of North \\ Carolina at Chapel Hill, Chapel Hill, \\ NC, USA; ${ }^{2}$ Durham VA Medical Center, \\ Durham, NC, USA; ${ }^{3}$ Department \\ of Medicine, Section of Pulmonary, \\ Critical Care, Allergy, and Immunology, \\ School of Medicine, Wake Forest \\ University, Winston-Salem, NC, USA
}

Purpose: Adequate peak inspiratory flow rate (PIFR) is required for drug dispersion with dry powder inhalers (DPIs). Prevalence of PIFR discordance (suboptimal PIFR with prescribed inhalers) and factors influencing device-specific PIFR are unclear in COPD. The objective of this study was to determine the prevalence of PIFR discordance and associated clinical factors in a stable COPD population.

Patients and methods: An observational, single-center, cohort study was conducted including 66 outpatients with COPD. PIFR was measured using the In-Check ${ }^{\mathrm{TM}}$ Dial with applied resistance of prescribed inhalers. Participants were defined as discordant if measured PIFR was $<30 \mathrm{~L} / \mathrm{min}$ and $<60 \mathrm{~L} / \mathrm{min}$ for high and low-medium resistance devices, respectively, using an inspiratory effort the participant normally used with their prescribed DPI.

Results: The median age of the COPD participants was 69.4 years, $92 \%$ were white and $47 \%$ were female. A total of $48 \%$ were using low-medium resistance DPIs (Diskus ${ }^{\circledR} /$ Ellipta $^{\circledR}$ ) and $76 \%$ used high-resistance DPI (Handihaler ${ }^{\mathbb{R}}$ ). A total of $40 \%$ of COPD participants were discordant to prescribed inhalers. Female gender was the only factor consistently associated with lower PIFR. Shorter height was associated with reduced PIFR for low-medium resistance $(r=0.44$; $P=0.01)$, but not high resistance $(r=0.20 ; P=0.16)$. There was no correlation between PIFR by In-Check ${ }^{\mathrm{TM}}$ dial and PIFR measured by standard spirometer.

Conclusion: PIFR is reduced in stable COPD patients, with female gender being the only factor consistently associated with reduced PIFR. Discordance with prescribed inhalers was seen in $40 \%$ of COPD patients, suggesting that many COPD patients do not generate adequate inspiratory force to overcome prescribed DPIs resistance in the course of normal use.

Keywords: pulmonary disease, chronic obstructive, dry powder inhaler, peak inspiratory flow rate, drug delivery systems

\section{Introduction}

Pharmacotherapy for the treatment of COPD largely focuses on maintenance inhaler therapies to reduce symptom burden and frequency of exacerbations. ${ }^{1}$ In the United States, there are $>15$ approved inhaled maintenance therapies for the different combinations of long-acting beta-agonists, long-acting muscarinic agents, and corticosteroids used in COPD. ${ }^{2}$ These medications can be delivered through four possible delivery systems: metered dose inhalers (MDIs), dry powder inhalers (DPIs), soft mist inhalers (SMIs), or nebulizers. Each delivery device has attributes designed to facilitate ease of patient use and effective drug delivery. MDIs, one of the most common types of delivery devices, provide consistent and reliable dosing through pressurized delivery, but require patient coordination, proper preparation prior to use, and in some instances use of reservoir device (ie, spacer) for effective delivery. SMIs provide consistent delivery in part because of a slower aerosol generation, ${ }^{3}$ but require proper preparation
Correspondence: M Bradley Drummond Division of Pulmonary Diseases and Critical Medicine, Department of Medicine, University of North Carolina at Chapel Hill, Marsico Hall Room 7207 CB\# 7248, 125 Mason Farm Road, Chapel Hill, NC 27599, USA

Tel + I 9199667054

Fax +19199665178

Email brad_drummond@med.unc.edu 
prior to use and coordination of inhalation. DPIs are breathactuated, thereby minimizing the need for hand-breath coordination required with MDI use. However, DPIs require a peak inspiratory flow rate (PIFR) to overcome the device's unique internal resistance to appropriately disaggregate and disperse the drug powder. The ability to generate optimal PIFR is a major requirement of successful DPI use.

PIFR is the maximal flow obtained during a forced inspiratory maneuver, either with or without resistance. It is proportional to maximal inspiratory pressure, ${ }^{4}$ and both are regularly measured without resistance during standard spirometry. Minimal flow rates are device-specific, related to the particular design characteristics of each device (primarily resistance), and the molecules (both drug and carrier) contained within. ${ }^{4-8}$ A PIFR value of $60 \mathrm{~L} / \mathrm{min}$ is generally accepted to be optimal for most devices. ${ }^{9,10}$ However, PIFR values $>60 \mathrm{~L} / \mathrm{min}$ can be associated with excessively turbulent flow and therefore poor lung deposition. Similarly, lung deposition decreases in a nonlinear fashion from 60 to $30 \mathrm{~L} / \mathrm{min}$ with PIFR $<30 \mathrm{~L} / \mathrm{min}$ associated with insufficient lung deposition in some devices..$^{9-11}$ Not only does each DPI have its own unique resistance and molecules, but each has its own unique optimal PIFR. ${ }^{8}$ Therefore, the sufficiency of PIFR range $30-60 \mathrm{~L} / \mathrm{min}$ remains debated..$^{9,10,12,13}$ Suboptimal PIFR, where the PIFR is below the minimal threshold for the device, can lead to inadequate drug disaggregation and insufficient distal airway drug deposition. The predominant demographic factors that have consistently been associated with a lower PIFR are patient effort, female gender, and older age. ${ }^{4,8-10,14,15}$ Spirometric factors such as FEV1 and FEV1\% predicted have not shown consistent correlation with PIFR, indicating that severity of lung function impairment alone cannot be used for device selection. ${ }^{9,14-17}$ The clinical importance of reduced PIFR has also been recently demonstrated in studies reporting an association between reduced PIFR and increased risk of acute exacerbations of COPD and readmissions. ${ }^{16,17}$

Despite extensive reviews showing the significance of age, gender, patient effort, and device resistance, ${ }^{4,8}$ much of the existing clinical literature describing factors and impact of suboptimal PIFR has focused on assessing PIFR without incorporating the specific DPI resistance into the measurement of PIFR. The In-Check ${ }^{\mathrm{TM}}$ Dial G16 (Alliance Tech, Granbury, TX, USA) is a hand-held device with an adjustable dial to impose resistance to mimic different inhaler types. ${ }^{18}$ Applying the device-specific resistance with the In-Check ${ }^{\mathrm{TM}}$ Dial permits the determination of PIFR discordance, defined as present when a patient has suboptimal PIFR specific to his or her prescribed inhaler. This study aimed to analyze an outpatient COPD population free of recent exacerbations to determine prevalence and clinical factors associated with PIFR discordance (PIFR mismatch with prescribed inhalers) using inhaler-specific resistance testing through the In-Check ${ }^{\mathrm{TM}}$ Dial with participants using inspiratory effort normally used to self-administer their prescribed DPI.

\section{Materials and methods Design}

We conducted an observational, single-center, prospective cohort study to determine the association between PIFR across a range of inhaler resistance profiles and clinical characteristics in a population of stable COPD patients. A subset of COPD participants returned for repeated measurements at 2-4 weeks to determine reproducibility of PIFR.

\section{Study participants}

The cohort for this study included sequential eligible patients recruited from the University of North Carolina (UNC) Outpatient Pulmonary Clinic between February 13, 2017 and April 30, 2018. All eligible participants had spirometryconfirmed COPD (defined as post-bronchodilator ratio of FEV1 to FVC $<0.70$ and FEV1/FVC $<$ lower limit of normal) within 1 year of screening. Participants were on stable maintenance inhaler COPD therapy with at least one DPI and free of acute exacerbations of COPD (defined as requiring steroids or antibiotics for worsening of pulmonary symptoms) in the 6 weeks prior to enrollment. Additional exclusion criteria included self-reported pregnancy. All participants provided written informed consent, and the study protocol was approved by the UNC Institutional Review Board and was conducted in accordance with the Declaration of Helsinki.

\section{Data collection}

Demographics and clinical data included age, race, gender, recent spirometry results (obtained within the prior year), and current inhaler use. The median time from spirometry testing to visit date was 63 days. Symptoms were assessed using the COPD Assessment Test (CAT). ${ }^{19}$

Peak inspiratory flow measurements were obtained with the In-Check ${ }^{\mathrm{TM}}$ Dial G16 (Alliance Tech). ${ }^{18}$ The In-Check $^{\mathrm{TM}}$ Dial is a hand-held device with disposable mouthpieces and an adjustable dial to impose resistance to mimic different inhaler types. Participants had PIFR measured with the resistance settings correlating to the DPIs they reported currently using. The resistances evaluated were low-medium resistance [mimicking Diskus ${ }^{\circledR}$ and Ellipta ${ }^{\circledR}$, heretofore termed "Resistance-2 (R2)"] and high resistance [mimicking 
Handihaler ${ }^{\circledR}$, heretofore termed "Resistance 5 (R5)"]. Participants were asked to inhale through the In-Check ${ }^{\mathrm{TM}}$ Dial TM "as if using your inhaler." Participants did not receive any education on inhaler technique during the study. Measurements were collected in triplicate for each device. Ample time was given between measurements to prevent fatigue. PIFR measurements were repeated in a subset of participants at 2-4 weeks in the context of this research protocol if study participants remained exacerbation free and were without a change in maintenance therapy.

\section{Statistical methods}

Summary measures for clinical and demographic characteristics are presented as median (IQR) or n (\%). Triplicate PIFR measurements were averaged at each resistance tested to obtain a mean value for each individual. Repeatability of PIFR measurements were assessed with Wilcoxon matched-pairs signed-rank test. Correlations between PIFR measurements and clinical characteristics were assessed with pairwise Pearson's correlation coefficients. Analyses were conducted at the patient level except when describing individual inhaler use frequencies or inhaler-level PIFR measurements.

Participants were stratified into discordant or concordant inhaler use based on PIFR measurement at the resistances of their prescribed R2 or R5 inhalers. We defined a participant as discordant if his/her measured PIFR $<60 \mathrm{~L} / \mathrm{min}$ on R2 device or PIFR $<30 \mathrm{~L} / \mathrm{min}$ on R5 device. If a participant was on both R2 and R5 devices, discordance was present if PIFR was suboptimal with either device. Concordant participants' PIFR measurements exceeded $30 \mathrm{~L} / \mathrm{min}$ for R5 and $60 \mathrm{~L} / \mathrm{min}$ for R2 for prescribed inhalers.

\section{Results}

\section{Participant characteristics}

The total cohort included 66 study participants prescribed DPIs (Table 1). The median age of COPD cohort was 69.4 years old, $92 \%$ were white and $47 \%$ were female. The median FEV1 of COPD participants was $1.35 \mathrm{~L}$ (51.3\% percent predicted). The median PIFR measured from standard spirometer among COPD participants was $172 \mathrm{~L} / \mathrm{min}$. The distribution of Global Initiative for Obstructive Lung Disease (GOLD) stages included $8 \%$ GOLD stage I, 45\% GOLD stage II, $35 \%$ GOLD stage III, and 12\% GOLD stage IV. The median CAT score was 16.5 with $88 \%$ of study participants having a score $\geq 10$. The most commonly used DPI was the $\mathrm{R} 5$ resistance device used by $76 \%$ of COPD participants, followed by the $\mathrm{R} 2$ resistance device used by $48 \%$ of the cohort (Table 2 ).
Table I Baseline demographics and clinical characteristics

\begin{tabular}{|l|l|}
\hline N & \multicolumn{1}{l|}{66} \\
\hline Age, years & $69.4(60-77)$ \\
\hline Race & $6 I(92)$ \\
\hline White & $4(6)$ \\
\hline African-American & $\mathrm{I}(2)$ \\
\hline Other & $3 \mathrm{I}(47)$ \\
\hline Female & $0.49(0.4 \mathrm{I}-0.58)$ \\
\hline FEVI/FVC ratio & $1.35(1.04-1.69)$ \\
\hline FEVI absolute, L & $51.3(4 \mathrm{I} .0-64.1)$ \\
\hline FEVI \% predicted & $2.80(2.27-3.57)$ \\
\hline FVC absolute, L & $78.4(70.8-94.0)$ \\
\hline FVC \% predicted & $172(12 \mathrm{I}-2 \mathrm{I} 5)$ \\
\hline PIFR from standard spirometer, L/min & \\
\hline GOLD stage & $5(8)$ \\
\hline I & $30(45)$ \\
\hline 2 & $23(35)$ \\
\hline 3 & $8(I 2)$ \\
\hline 4 & $16.5(12-24)$ \\
\hline CAT score & $58(88)$ \\
\hline CAT score $\geq I 0$ &
\end{tabular}

Note: All values are given as $\mathrm{n}(\%)$ or median (IQR).

Abbreviations: GOLD, Global Initiative for Obstructive Lung Disease; CAT, COPD assessment test; PIFR, peak inspiratory flow rate.

Only one participant used solely an R3 resistance device, while none had either R1 or R4 DPIs, and thus these resistance levels were not used in the concordance analysis.

\section{PIFR measurements and repeatability in COPD}

We assessed PIFR in COPD participants after applying the resistance of the two most prevalent DPI resistance profiles (R2 and R5). For R2 resistance, the median (IQR) PIFR was 64 (43-74) L/min. For R5 resistance, COPD participants had a median (IQR) PIFR of 36 (28-39) L/min. Figure 1 shows the distribution of participants within PIFR bins $(<30,31-45$, 46-60, and $>60$ ) with R2 and R5 measurements. PIFR $<60$ $\mathrm{L} / \mathrm{min}$ was present with $44 \%$ of R2 inhaler use, and PIFR $<30 \mathrm{~L} /$ min was present with $32 \%$ of R5 inhaler use. When stratifying by GOLD FEV1 stage (Figure 2), participants with suboptimal PIFR were distributed as follows: R2 inhalers (PIFR $<60 \mathrm{~L} / \mathrm{min}$ ), $7 \%$ stage I, 57\% stage II, 36\% stage III; R5 inhalers (PIFR $<30 \mathrm{~L} / \mathrm{min}$ ), $0 \%$ stage I, $56 \%$ stage II, $31 \%$ stage III, $2 \%$ stage IV.

A total of 15 COPD participants underwent repeated measures at 2-4 weeks (Figure 3 and Table S1). Among the COPD patients, there was a statistically significant difference in the overall distribution $(P=0.04)$, with second 
Table 2 Inhaler prescriptions

\begin{tabular}{|c|c|}
\hline $\begin{array}{l}\text { Prescribed inhaler (resistance class and } \\
\text { representative inhalers) }\end{array}$ & $\begin{array}{l}\text { Number } \\
\text { of COPD } \\
\text { patients, } n(\%)^{b}\end{array}$ \\
\hline $\begin{array}{l}\text { RI } \\
\text { - Breezhaler }{ }^{\circledR}\left(\text { Seebri }^{\circledR}\right) \\
\text { - } \text { Neohaler }^{\circledR}\left(\text { Utibron }^{\circledR}\right)\end{array}$ & $0(0)$ \\
\hline $\begin{array}{l}\text { R2 } \\
\text { - } \text { Diskus }^{\circledR}\left(\text { eg, } \text { Advair }^{\circledR}, \text { Serevent }\right. \\
\text { - } \text { Ellipta }^{\circledR}\left(\text { eg, } \text { Anoro }^{\circledR}, \text { Breo }^{\circledR}, \text { Incruse }^{\circledR}, \text { Trelegy }^{\circledR}\right)\end{array}$ & $32(48)$ \\
\hline $\begin{array}{l}\text { R3 } \\
\text { - } \text { Pressair }^{\circledast}\left(\text { Tudorza }^{\circledR}\right) \\
\text { - Genuair } \\
\text { - } \text { - }^{\circledR} \text { clidinium } \\
\text { - }\end{array}$ & $2(3)$ \\
\hline $\begin{array}{l}\text { R4 } \\
\text { - Turbuhaler }{ }^{\circledast}\left(\text { Pulmicort }^{\circledast}\right) \\
\text { - Twistihaler } \\
\left(\text { Asmanex }^{\circledast}\right)\end{array}$ & $0(0)$ \\
\hline $\begin{array}{l}\text { R5 } \\
\text { - Handihaler }{ }^{\circledast}\left(\text { Spiriva }^{\circledast}\right)\end{array}$ & $50(76)$ \\
\hline
\end{tabular}

Notes: " " $R$ " refers to resistance class of inhaler, with RI being the lowest resistance and R5 being the highest resistance device. See "Materials and methods" section for additional explanation. 'Column total sums to $>66$ as participants could be on multiple inhalers. All values are given as $\mathrm{n}(\%)$.

measurements being higher. The six COPD participants using $\mathrm{R} 2$ inhalers did not have a difference in PIFR measurements at baseline vs follow-up $(65 \mathrm{~L} / \mathrm{min}$ on visit 1 and $64 \mathrm{~L} / \mathrm{min}$ on visit 2; $P=0.69)$. Of the 14 participants on an $\mathrm{R} 5$ inhaler, there was no difference in baseline and repeated PIFR measurement $(38 \mathrm{~L} / \mathrm{min}$ on visit 1 and $38 \mathrm{~L} / \mathrm{min}$ on visit $2 ; P=0.46)$.

\section{Factors associated with lower PIFR}

The correlation between clinical factors and PIFR measurement was evaluated. Factors evaluated included age, sex, FEV1, peak inspiratory flow from spirometry measurements, FVC, CAT score, height, and body mass index (BMI).

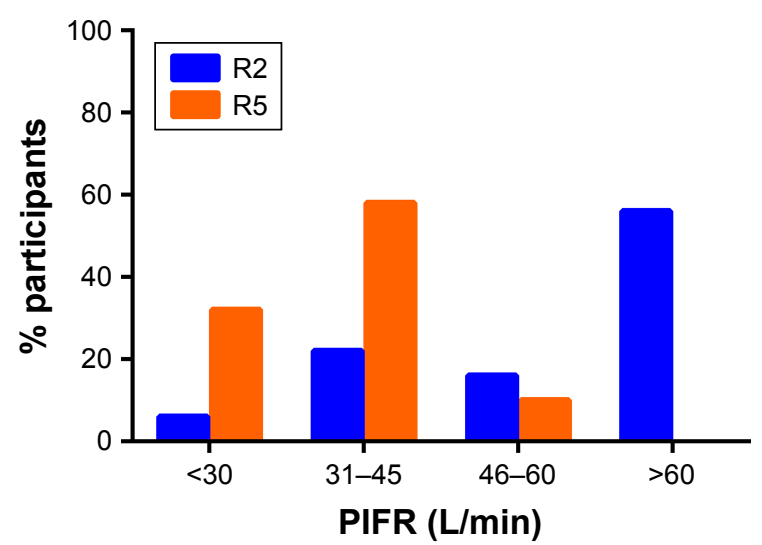

Figure I Percentage of patients within bins of peak inspiratory flow (PIFR), measured against R2 low-medium resistance inhaler (eg, Diskus ${ }^{\circledR}$ and Ellipta ${ }^{\circledR}$; blue) and R5 high resistance inhaler (Handihaler ${ }^{\circledR}$; orange).

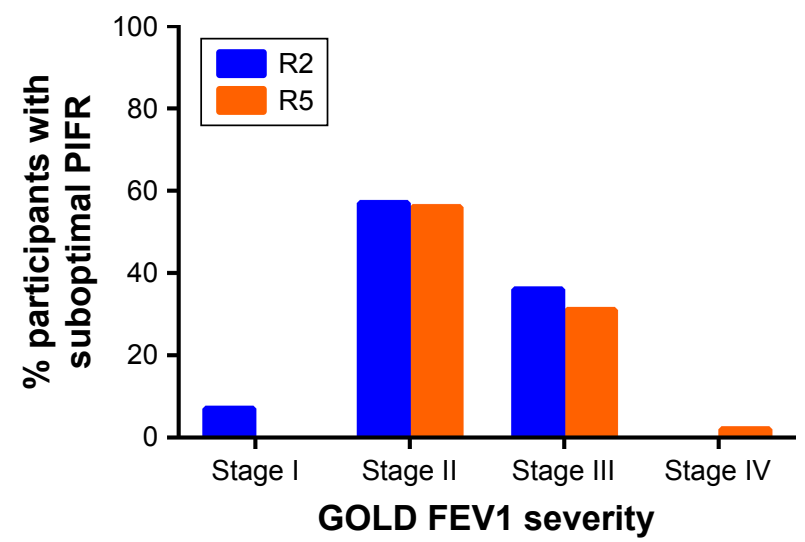

Figure 2 Distribution of patients with suboptimal peak inspiratory flow (PIFR) stratified by Global Initiative for Obstructive Lung Disease (GOLD) FEVI severity stage, measured against R2 low-medium resistance inhaler (eg, Diskus ${ }^{\circledR}$ and Ellipta ${ }^{\circledR}$; blue; suboptimal PIFR $<60 \mathrm{~L} / \mathrm{min}$ ) and $\mathrm{R} 5$ high resistance inhaler (Handihaler ${ }^{\circledR}$; orange; suboptimal PIFR $<30 \mathrm{~L} / \mathrm{min})$.

The only factor found to be consistently associated with a lower PIFR was female gender. For R2 resistance, the median (IQR) PIFR was 49.7 L/min (40.3-64.7 L/min) for women compared to $70.7 \mathrm{~L} / \mathrm{min}(57-75 \mathrm{~L} / \mathrm{m}$ in) for men $(P=0.03)$ (Figure $\mathrm{S} 1)$. For R5 resistance, the median (IQR) PIFR was $35.2 \mathrm{~L} / \mathrm{min}(23.5-37.5 \mathrm{~L} / \mathrm{min})$ for women compared to $37.5 \mathrm{~L} / \mathrm{min}(28-42.1 \mathrm{~L} / \mathrm{min})$ for men $(P=0.042)$ (Figure S2). Shorter height was associated with reduced PIFR for R2 resistance $(r=0.44 ; P=0.01)$ but not for R5 resistance $(r=0.20 ; P=0.16)$ (Figures S3 and S4). PIFR measured against R2 and R5 resistance did not correlate with the PIFR measured by standard spirometer $(r=0.05[P=0.80]$ and $r=0.10[P=0.53]$, respectively) (Figures S5 and S6). For the $\mathrm{R} 2$ resistance there was no correlation with PIFR and age $(r=0.14 ; P=0.44)$, CAT score $(r=0.17 ; P=0.35)$, FEV1 $(r=-0.28 ; P=0.12)$, FVC $(r=0.08 ; P=0.66)$, or BMI $(r=0.10$; $P=0.61)$. For the $\mathrm{R} 5$ resistance there was also no correlation

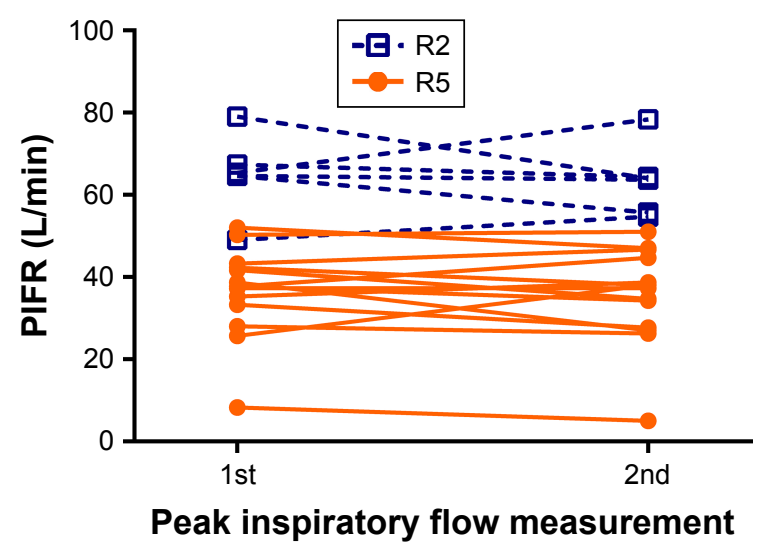

Figure 3 Repeatability of peak inspiratory flow rate (PIFR) from baseline to 2-4 weeks, measured against R2 low-medium resistance inhaler (eg, Diskus ${ }^{\circledR}$ and Ellipta ${ }^{\circledR}$; blue dashed line) and R5 high resistance inhaler (Handihaler ${ }^{\circledR}$; orange solid line). 
with PIFR and age $(r=0.06 ; P=0.70)$, CAT score $(r=0.22$; $P=0.13)$, FEV1 $(r=0.03 ; P=0.82)$, FVC $(r=0.06 ; P=0.69)$, or BMI $(r=0.07 ; P=0.65)$.

\section{Discordance between prescribed inhaler and PIFR}

A participant was defined as discordant if he or she had a PIFR $<60 \mathrm{~L} / \mathrm{min}$ on $\mathrm{R} 2$ device or PIFR $<30 \mathrm{~L} / \mathrm{min}$ on $\mathrm{R} 5$ device. If a participant was on both R2 and R5 devices, discordance was present is PIFR was suboptimal on either device. Of 65 total study participants on R2 and/or R5 devices (one participant on R3 device only and thus excluded for discordance analyses), 39 (60\%) were concordant with inhalers (Table 3). Among the 26 discordant participants, the median age was 72.1 years, $85 \%$ were white, $62 \%$ were female, and the median FEV1 was $56.0 \%$ predicted. Concordant participants had an average age of 66.4 years, $97 \%$ were white, $39 \%$ were female, and the average FEV1 was $48.5 \%$ predicted. The CAT score was elevated in concordant and discordant participants, with $>75 \%$ in both groups having CAT scores $\geq 10$. There were no clinical differences between concordant and discordant groups. The prevalence of use of a high resistance DPI was not different between discordant and concordant participants. Similarly, use of a low-medium resistance DPI did not differ between discordant and concordant participants.

\section{Discussion}

In this analysis of 66 participants with stable COPD in the outpatient setting, we have found that PIFR was reduced to $<60 \mathrm{~L} / \mathrm{min}$ among $\sim 45 \%$ of COPD individuals when applying low-medium resistance and $\sim 30 \%$ of COPD individuals when applying high resistance to the In-Check ${ }^{\mathrm{TM}}$ Dial. This reduction was persistent over repeated measures. Confirming prior reports, female gender was a clinical factor consistently associated with reduced PIFR in COPD. There was no correlation between PIFR measured with spirometry and PIFR measured against resistance using the In-Check ${ }^{\mathrm{TM}}$ Dial, highlighting the challenge of predicting reduced PIFR in COPD. Discordance, defined as suboptimal PIFR against any prescribed inhaler, was observed in $40 \%$ of COPD patients without the commonly reported factors associated with low PIFR such as poor effort, older age, small stature, malnutrition, and air trapping. ${ }^{4,8}$

The increased prevalence of discordance with any one prescribed inhaler highlights that many COPD patients do not generate adequate inspiratory force to overcome the resistance of their prescribed inhalers. To our knowledge, this is the first study evaluating PIFR applying the resistance of DPIs specific to the participant's inhalers prescribed in clinical practice, with inspiratory effort reflecting the participant's normal effort. In our study, discordance was

Table 3 Demographics, clinical characteristics, and DPI use in discordant vs concordant participants

\begin{tabular}{|c|c|c|c|}
\hline & Concordant & Discordant & $P$-value \\
\hline N (\%) & $39(60)$ & $26(40)$ & \\
\hline Age, years & $66.4(58-76)$ & $72.1(64-80)$ & 0.56 \\
\hline White race & $38(97)$ & $22(85)$ & 0.13 \\
\hline Female & 15 (39) & $16(62)$ & 0.08 \\
\hline Height, cm & 173 (I57-I78) & 164 (I57-I73) & 0.19 \\
\hline BMI $\left(\mathrm{kg} / \mathrm{m}^{2}\right)$ & $27(25-34)$ & $28(23-3 I)$ & 0.79 \\
\hline FEVI/FVC ratio & $0.47(0.37-0.59)$ & $0.5 \mathrm{I}(0.44-0.55)$ & 0.77 \\
\hline FEVI absolute, $\mathrm{L}$ & $1.33(1.04-1.67)$ & $1.32(0.97-1.74)$ & 0.33 \\
\hline FEVI \% predicted & $48.5(39-57)$ & $56.0(44-66)$ & 0.76 \\
\hline FVC absolute, L & $2.85(2.45-3.59)$ & $2.59(2.05-3.33)$ & 0.36 \\
\hline FVC \% predicted & 76.9 (69-94) & $83.0(74-97)$ & 0.92 \\
\hline PIFR from standard spirometer, L/min & $170(|2|-2 \mid 5)$ & $167(121-210)$ & 0.80 \\
\hline GOLD stage & & & 0.32 \\
\hline 1 & $4(11)$ & I (4) & \\
\hline 2 & $14(36)$ & $15(58)$ & \\
\hline 3 & $15(38)$ & $8(31)$ & \\
\hline 4 & $6(15)$ & $2(8)$ & \\
\hline CAT score & $16(12-23)$ & $18(11-25)$ & 0.65 \\
\hline CAT score $\geq 10$ & $37(95)$ & $20(77)$ & 0.51 \\
\hline Low-medium resistance DPI & $17(44)$ & $15(58)$ & 0.27 \\
\hline High resistance DPI & $32(82)$ & $18(69)$ & 0.23 \\
\hline
\end{tabular}

Notes: All values are either $\mathrm{n}(\%)$ or median (IQR). See (Materials and Methods) section for information regarding definition of discordance. P-value via Kruskal-Wallis for continuous variables and Fisher's exact test for categorical variables.

Abbreviations: DPI, dry powder inhaler; CAT, COPD assessment test; BMI, body mass index; GOLD, Global Initiative for Obstructive Lung Disease. 
present among both low-medium and high resistance DPIs, indicating that the majority of those prescribed DPIs are at risk for not obtaining appropriate drug dispersion of their maintenance inhalers. One study performed PIFR using the InCheck ${ }^{\mathrm{TM}}$ Dial device in ambulatory patients after performing spirometry using the Diskus ${ }^{\circledR}$ resistance. ${ }^{20}$ Twenty-four percent of the 71 COPD patients did not achieve a PIFR $\geq 60 \mathrm{~L} / \mathrm{min}$. In our study, discordance remained prevalent when using device-specific cutoffs of $30 \mathrm{~L} / \mathrm{min}$ for R5 and $60 \mathrm{~L} / \mathrm{min}$ for R2.

The importance of suboptimal PIFR has been made clear by studies reporting an association between reduced PIFR and increased risk of acute exacerbations of COPD and readmissions. ${ }^{16,17}$ Measurement of PIFR allows providers to identify which participants should be switched to a lower resistance DPI, MDI, SMI, or nebulizer. While this study focused on the stable outpatient setting, PIFR appears to be even lower during an exacerbation, ${ }^{17}$ and further research is required to identify how PIFR and DPI use is affected during exacerbations.

Many studies have examined correlations between lower PIFR and demographic factors. Our results are consistent with prior findings that female gender has been associated with a lower PIFR. ${ }^{14-16,21}$ While our analysis did not show a correlation between age and PIFR, older age has been repeatedly associated with a lower PIFR in other studies. ${ }^{9} 14,15,17,22-24$ Our study had a narrow range of age, hence we did not see an age association. Shorter stature was associated with reduced PIFR when measured against a low-medium resistance setting, but not high resistance. The association between shorter stature and reduced PIFR with simulated resistance of Diskus ${ }^{\circledR}$ has previously been reported. ${ }^{14}$ Nevertheless, while one should have a higher index suspicion of lower PIFR among female patients or those of shorter stature, measuring PIFR is the only reliable method to know which patients will have a low PIFR.

As has been described previously and re-demonstrated here, severity of obstruction is not consistently associated with low PIFR, except perhaps in those with FEV1 $<30 \%$ predicted. ${ }^{25}$ Low PIFR may be a measurement of hyperinflation as Mahler et al have described an association with lower PIFR and FVC, ${ }^{14}$ with FVC observations also reported by Tung et al. ${ }^{20}$ The population studied by Mahler had more severe obstruction than our study population, highlighting that severe COPD may be associated with differential impacts on PIFR than mild and moderate COPD. While standard spirometry does include measurement of PIFR, this measurement does not incorporate resistance of the inhaler device.
Dewar et al observed a weak correlation between the PIFR obtained through a Turbuhaler ${ }^{\circledR}$ resistance and standard spirometry $(r=0.53)$. This correlation, however, was not strong enough to determine a spirometric PIFR cutoff value which would be sufficient for a Turbuhaler ${ }^{\circledR}{ }^{13}$ Seheult et al observed a stronger correlation between spirometric PIFRs, obtained without resistance and with Diskus ${ }^{\circledR}$ PIFR, demonstrating that all participants with a spirometric PIFR $>120 \mathrm{~L} / \mathrm{min}$ were able to reach the minimum required PIFR for Diskus ${ }^{\circledR}{ }^{26}$ Both of these studies were device-specific, and not generalizable to all-comers in the clinical setting. Our data showed no correlation with spirometric PIFR and R2 (ie, Diskus ${ }^{\circledR}$ ) or R5 (ie, Handihaler ${ }^{\circledR}$ ), indicating that there are likely multiple factors contributing to a resistance-specific PIFR.

Few studies have shown reproducibility of PIFR measurements over time in stable outpatients. Broeders et al primarily studied the effect of instruction on optimizing PIFR; they however also found that PIFR measurements were reproducible within same day measurements with a 4\%-10\% variability. ${ }^{27}$ Among COPD participants, we found no difference in PIFR in R2 or R5 resistances measured 2-4 weeks after initial measurements, indicating that PIFR is a reproducible measurement in stable populations.

This study has limitations. It was a single-center cohort. The study cohort was predominantly Caucasian, and few had very severe airflow obstruction, which may limit generalizability. The study sample may have been under-powered to detect an association between PIFR, risk factors such as age and height, and symptom burden. Larger studies with populations containing more heterogeneous characteristics (eg, ethnicity, socioeconomic status, and health literacy) are required to definitely determine an association between PIFR, risk factors, and symptom burden. In this study, participants were given no instruction on how to optimize PIFR, which may impact our results. We chose to evaluate mean PIFR over three measurements, but an alternative approach is to use maximum of three measurements to define PIFR. We chose mean PIFR as we feel this best attenuates any training or fatigue effect that may occur in triplicate measures. In our study, the median difference between maximum and mean PIFR was $3.6 \mathrm{~L} / \mathrm{min}$ for $\mathrm{R} 2$ and $2.0 \mathrm{~L} / \mathrm{min}$ for R5. When categorizing discordance using mean compared to maximum PIFR, 61 (94\%) of participants' categorization remained unchanged. Thus, we found minimal difference between definition of PIFR using maximum or mean of three measures.

Despite its limitations, this study represents an important contribution to our in vivo knowledge relative to PIFR. 
It highlights an important concept; suboptimal PIFR is a relative construct, impacted by device design and contents. Therefore, suboptimal PIFR for one device does not predict suboptimal PIFR for another device and thus a patient who receives an inadequate dose of drug from one device may be able to use another device adequately. To our knowledge this study is one of the first studies to look at inhaler discordance in a real-world setting specifically by asking study participants to inhale as if using their prescribed inhaler. There was a wide variability in technique, and given these inhalations were witnessed, PIFRs may be potentially lower at home. Given the prevalence of discordance to prescribed inhalers, this highlights a potential area of future research and focus for clinical improvement. What remains unclear is whether optimizing PIFR, therefore drug delivery, is modifiable. ${ }^{27}$

\section{Conclusion}

In conclusion, in this observational, single-center, cohort study, we found that PIFR is reduced, persistent, and associated with female gender in stable COPD patients. Discordance with prescribed inhalers is common. This study highlights that predicting suboptimal PIFR from clinical characteristics is challenging, and direct measure of PIFR may be a useful clinical tool for providers to optimize therapy in COPD patients prescribed DPIs.

\section{Acknowledgments}

MBD was supported by R01HL125432-01A1 and SG was supported by T32HL007106-41. MBD had full access to all of the data in the study.

\section{Author contributions}

All authors contributed to data analysis, drafting and revising the article, gave final approval of the version to be published, and agree to be accountable for all aspects of the work.

\section{Disclosure}

RAP has research support from Boehringer-Ingelheim and GlaxoSmithKline, served on Advisory Board for GlaxoSmithKline and Teva, has served as speaker for AstraZeneca and Sunovion, and served on Data Safety Monitoring Board for Grifols. JAO has performed advisory consulting for Boehringer-Ingelheim, GlaxoSmithKline, Theravance, Novartis, and AstraZeneca. She also has an investigator-initiated research grant from Sunovion. JFD has served as advisor to AZ, GSK, Novartis, Mylan, Sunovion, and Theravance. MBD has performed advisory consulting for
Boehringer-Ingelheim, GlaxoSmithKline, and AstraZeneca. There were no funders, thus no funders had a role in the design of the study; in the collection, analyses, or interpretation of data; in the writing of the manuscript; and in the decision to publish the results. SG reports no conflicts of interest in this work.

\section{References}

1. Global strategy for prevention, diagnosis, and management of COPD; 2018. Available from: www.goldcopd.org. Accessed November 5, 2018.

2. FDA approved drugs for pulmonary/respiratory diseases. Available from: https://www.centerwatch.com/drug-information/fda-approveddrugs/therapeutic-area/18/pulmonary-respiratory-diseases. Accessed November 5, 2018.

3. Pitcairn G, Reader S, Pavia D, Newman S. Deposition of corticosteroid aerosol in the human lung by Respimat soft mist inhaler compared to deposition by metered dose inhaler or by Turbuhaler dry powder inhaler. $J$ Aerosol Med. 2005;18(3):264-272.

4. Clark AR, Hollingworth AM. The relationship between powder inhaler resistance and peak inspiratory conditions in healthy volunteers implications for in vitro testing. J Aerosol Med. 1993;6(2):99-110.

5. Ghosh S, Ohar JA, Drummond MB. Peak inspiratory flow rate in chronic obstructive pulmonary disease: implications for dry powder inhalers. J Aerosol Med Pulm Drug Deliv. 2017;30(6):381-387.

6. Haidl P, Heindl S, Siemon K, Bernacka M, Cloes RM. Inhalation device requirements for patients' inhalation maneuvers. Respir Med. 2016; 118:65-75.

7. Finlay WH, Martin AR. Modeling of aerosol deposition with interface devices. J Aerosol Med. 2007;20(Supp1 1):S19-S28. discussion S27-S18.

8. Clark AR. The role of inspiratory pressures in determining the flow rates though dry powder inhalers; a review. Curr Pharm Des. 2015;21(27): 3974-3983.

9. Janssens W, Vandenbrande P, Hardeman E, et al. Inspiratory flow rates at different levels of resistance in elderly COPD patients. Eur Respir J. 2008;31(1):78-83.

10. Mahler DA. Peak inspiratory flow rate as a criterion for dry powder inhaler use in chronic obstructive pulmonary disease. Ann Am Thorac Soc. 2017;14(7):1103-1107.

11. Yokoyama H, Yamamura Y, Ozeki T, Iga T, Yamada Y. Analysis of relationship between peak inspiratory flow rate and amount of drug delivered to lungs following inhalation of fluticasone propionate with a Diskhaler. Biol Pharm Bull. 2007;30(1):162-164.

12. Chrystyn $\mathrm{H}$. Is inhalation rate important for a dry powder inhaler? Using the In-Check dial to identify these rates. Respir Med. 2003;97(2):181-187.

13. Dewar MH, Jamieson A, Mclean A, Crompton GK. Peak inspiratory flow through Turbuhaler in chronic obstructive airways disease. Respir Med. 1999;93(5):342-344.

14. Mahler DA, Waterman LA, Gifford AH. Prevalence and COPD phenotype for a suboptimal peak inspiratory flow rate against the simulated resistance of the Diskus ${ }^{\circledR}$ dry powder inhaler. J Aerosol Med Pulm Drug Deliv. 2013;26(3):174-179.

15. Malmberg LP, Rytilä P, Happonen P, Haahtela T. Inspiratory flows through dry powder inhaler in chronic obstructive pulmonary disease: age and gender rather than severity matters. Int $J$ Chron Obstruct Pulmon Dis. 2010;5:257-262.

16. van der Palen J. Peak inspiratory flow through diskus and turbuhaler, measured by means of a peak inspiratory flow meter (In-Check dial). Respir Med. 2003;97(3):285-289.

17. Loh CH, Peters SP, Lovings TM, Ohar JA. Suboptimal inspiratory flow rates are associated with chronic obstructive pulmonary disease and all-cause readmissions. Ann Am Thorac Soc. 2017;14(8):1305-1311.

18. Sanders MJ. Guiding inspiratory flow: development of the In-Check dial G16, a tool for improving inhaler technique. Pulm Med. 2017; 2017:1-7. 
19. Jones PW, Harding G, Berry P, Wiklund I, Chen WH, Kline Leidy N. Development and first validation of the COPD assessment test. Eur Respir J. 2009;34(3):648-654.

20. Tung LL, Duarte A, Mills J, Martinez J, Sharma G. Prevalence of low peak inspiratory flow rate in ambulatory patients with COPD. Am J Respir Critic Care Med. 2017;195:A5674.

21. Sharma G, Mahler DA, Mayorga VM, Deering KL, Harshaw O, Ganapathy V. Prevalence of low peak inspiratory flow rate at discharge in patients hospitalized for COPD exacerbation. Chronic Obstr Pulm Dis. 2017;4(3):217-224.

22. Seheult JN, Costello $\mathrm{S}$, Tee $\mathrm{KC}$, et al. Investigating the relationship between peak inspiratory flow rate and volume of inhalation from a Diskus $^{\mathrm{TM}}$ inhaler and baseline Spirometric parameters: a cross-sectional study. Springerplus. 2014;3:496.

23. Sharma GG, Mahler DA, Mayorga V, Deering K, Ganapathy V. Prevalence of Low Peak Inspiratory Flow Rate (PIFR) in Chronic Obstructive Pulmonary Disease (COPD) Patients at Discharge After an Exacerbation - Interim Findings from a Prospective Study. Amer J Resp Crit Care Med. 2016;193:A1506.
24. Kawamatawong T, Khiawwan S, Pornsuriyasak P. Peak inspiratory flow rate measurement by using In-Check dial for the different inhaler devices in elderly with obstructive airway diseases. J Asthma Allergy. 2017;10:17-21.

25. Prime D, de Backer W, Hamilton M, et al. Effect of disease severity in asthma and chronic obstructive pulmonary disease on Inhaler-Specific inhalation profiles through the ELLIPTA ${ }^{\circledR}$ dry powder inhaler. J Aerosol Med Pulm Drug Deliv. 2015;28(6):486-497.

26. Seheult JN, O'Connell P, Tee KC, et al. The acoustic features of inhalation can be used to quantify aerosol delivery from a Diskus ${ }^{\mathrm{TM}}$ dry powder inhaler. Pharm Res. 2014;31(10):2735-2747.

27. Broeders ME, Molema J, Hop WC, Folgering HT. Inhalation profiles in asthmatics and COPD patients: reproducibility and effect of instruction. J Aerosol Med. 2003;16(2):131-141. 


\section{Supplementary materials}

Table SI Repeatability of PIFR in COPD participants

\begin{tabular}{l|l|l|l}
\hline & First measure & Second measure & $P$-value \\
\hline R0 $(\mathrm{N}=15)$ & $>120(59$ to $>120)$ & $>120(72$ to $>120)$ & 0.04 \\
R2 $(\mathrm{N}=6)$ & $65(65-67)$ & $64(56-64)$ & 0.69 \\
R5 $(\mathrm{N}=14)$ & $38(33-42)$ & $38(28-45)$ & 0.46 \\
\hline
\end{tabular}

Notes: All values are median (IQR). R0= zero resistance, $\mathrm{R} 2=$ low-medium resistance, $\mathrm{R} 5=$ high resistance. See "Materials and methods" section and Table 2 for additional explanation.

Abbreviation: PIFR, peak inspiratory flow rate.

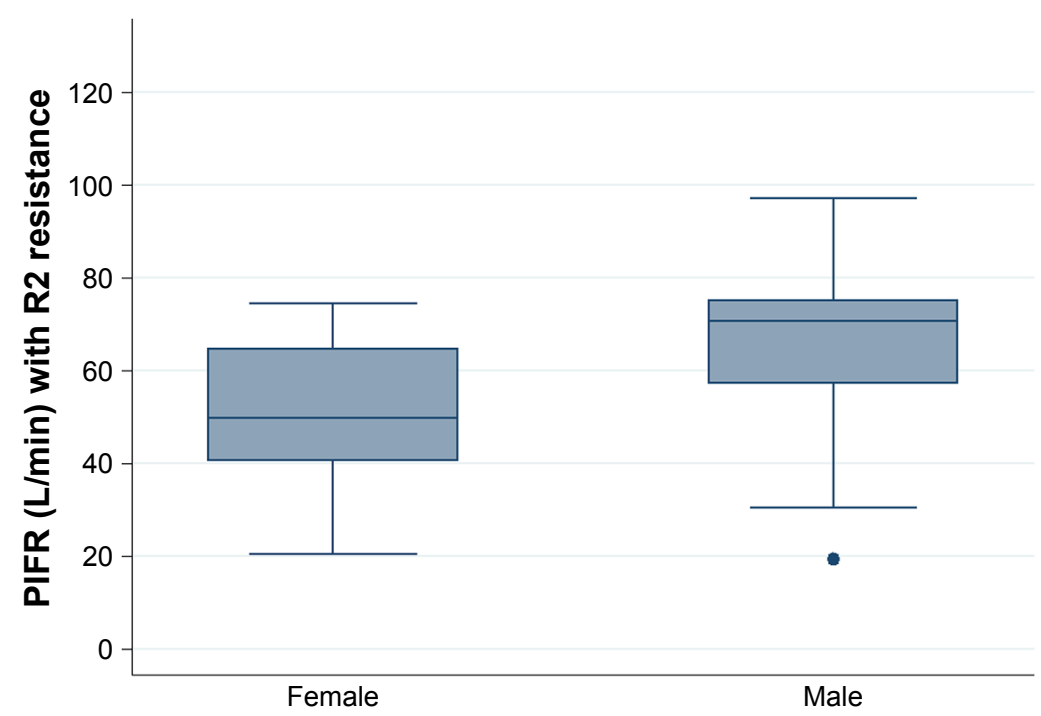

Figure SI Distribution of peak inspiratory flow rate (PIFR) between female and male participants when measured against R2 low-medium resistance inhaler (eg, Diskus ${ }^{\circledR}$ and Ellipta $\left.^{\circledR}\right)$.

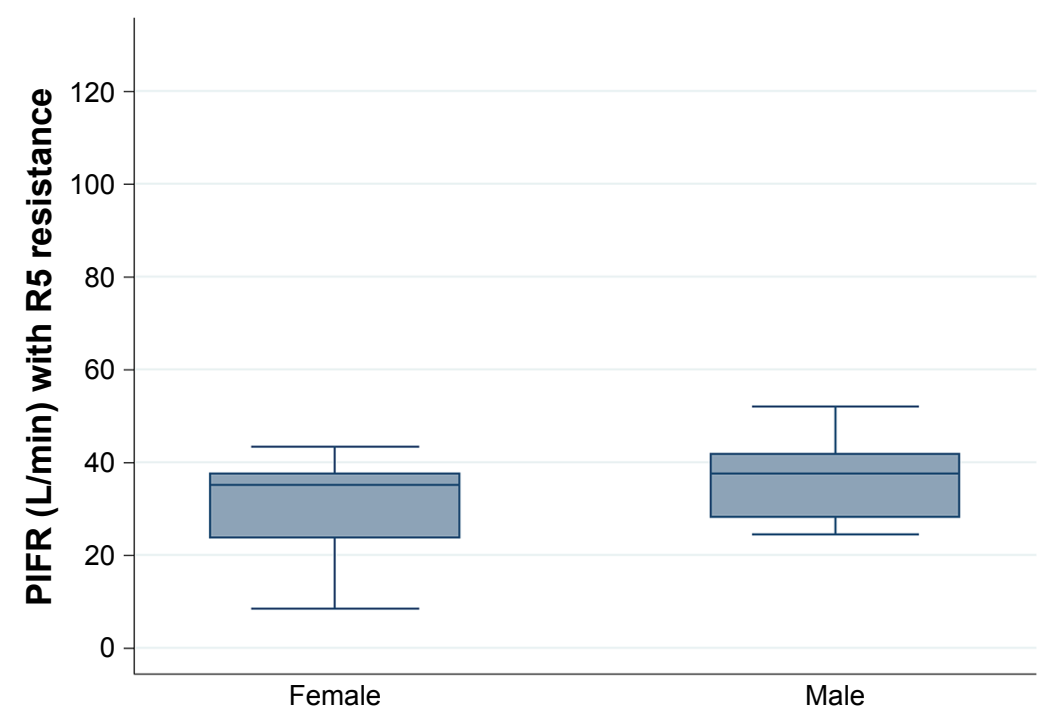

Figure S2 Distribution of peak inspiratory flow rate (PIFR) between female and male participants when measured against R5 high resistance inhaler (Handihaler ${ }^{\circledR}$ ). 


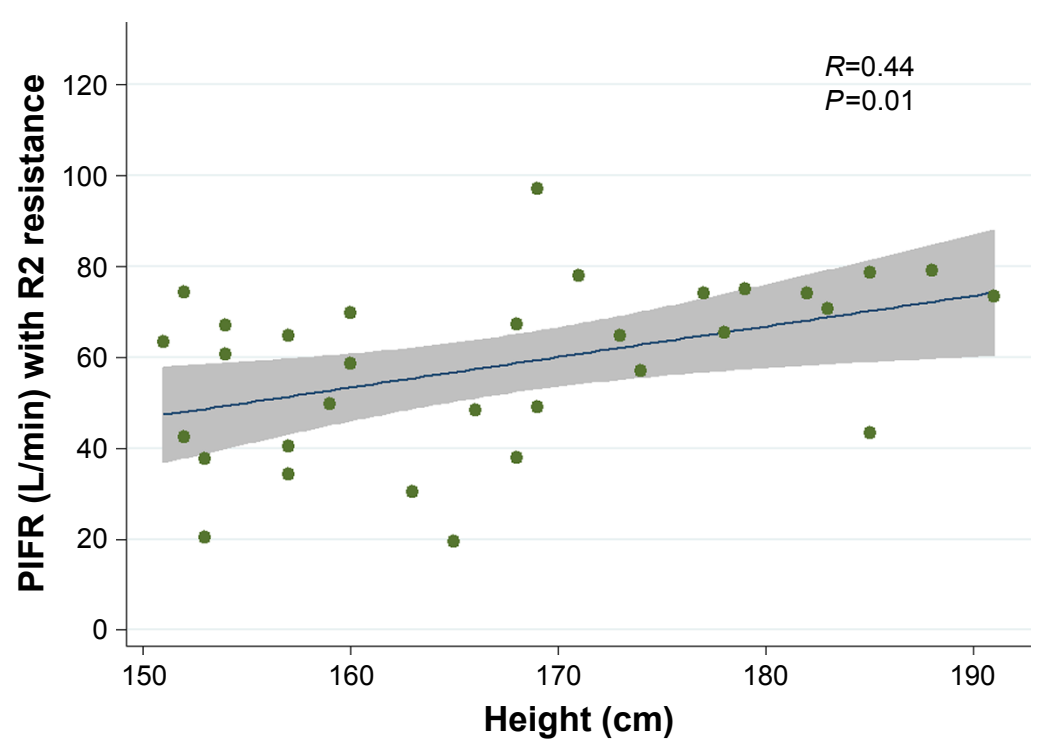

Figure S3 Correlation between height (x-axis) and In-Check ${ }^{\mathrm{TM}}$ Dial (y-axis) with R2 low-medium resistance profile inhaler (eg, Diskus ${ }^{\circledR}$ and Ellipta ${ }^{\circledR}$ ). Blue line represents fitted line with gray shading $95 \% \mathrm{Cl}$ of fitted line.

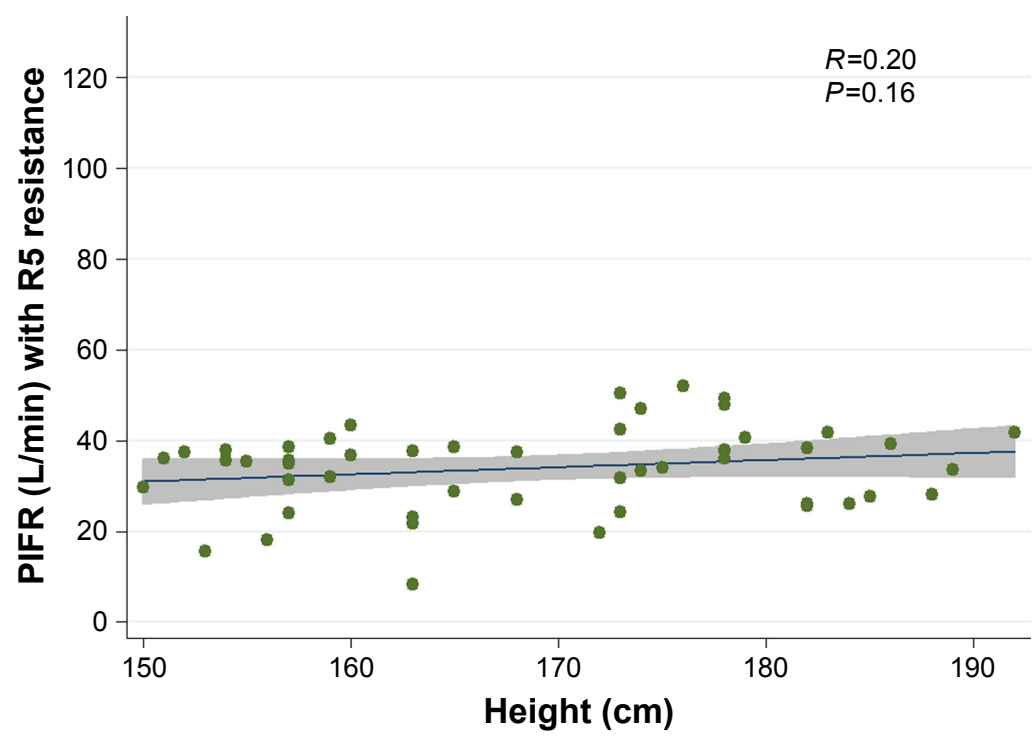

Figure S4 Correlation between height (x-axis) and In-Check ${ }^{\mathrm{TM}}$ Dial (y-axis) with R5 high resistance inhaler (Handihaler $\left.{ }^{\circledR}\right)$. Blue line represents fitted line with gray shading $95 \% \mathrm{Cl}$ of fitted line. 


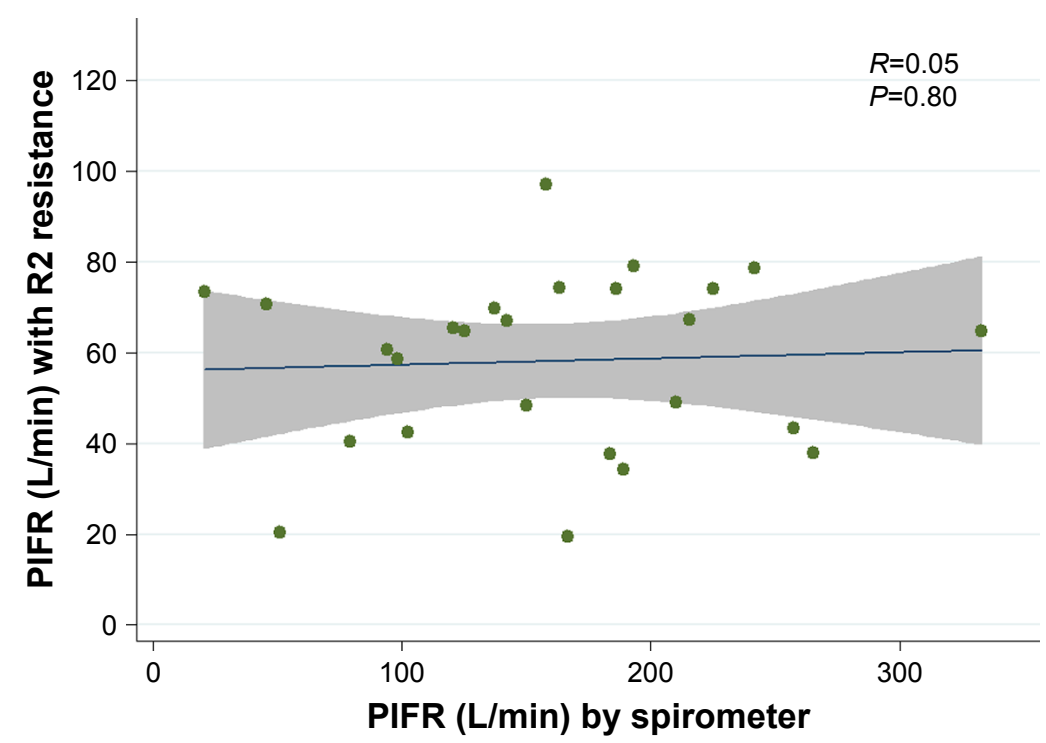

Figure S5 Correlation between peak inspiratory flow rate measured by standard spirometer ( $x$-axis) and In-Check ${ }^{\mathrm{TM}}$ Dial (y-axis) with R2 low-medium resistance profile inhaler (eg, Diskus ${ }^{\circledR}$ and Ellipta ${ }^{\oplus}$ ). Blue line represents fitted line with gray shading $95 \% \mathrm{Cl}$ of fitted line.

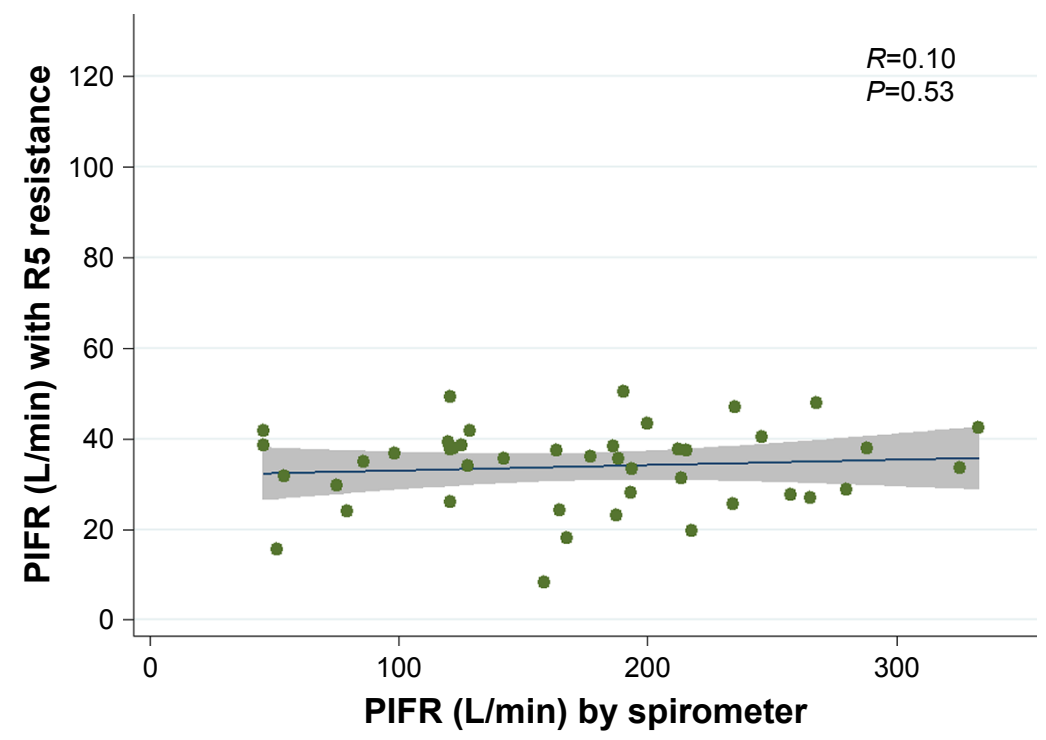

Figure S6 Correlation between peak inspiratory flow rate (PIFR) measured by standard spirometer ( $x$-axis) and In-Check ${ }^{\mathrm{TM}}$ Dial ( $y$-axis) with R5 high resistance profile inhaler (Handihaler $\left.{ }^{\circledast}\right)$. Blue line represents fitted line with gray shading $95 \% \mathrm{Cl}$ of fitted line.

International Journal of COPD

\section{Publish your work in this journal}

The International Journal of COPD is an international, peer-reviewed journal of therapeutics and pharmacology focusing on concise rapid reporting of clinical studies and reviews in COPD. Special focus is given to the pathophysiological processes underlying the disease, intervention programs, patient focused education, and self management protocols.

This journal is indexed on PubMed Central, MedLine and CAS. The manuscript management system is completely online and includes a very quick and fair peer-review system, which is all easy to use. Visit $\mathrm{http}: / / \mathrm{www}$.dovepress.com/testimonials.php to read real quotes from published authors.

Submit your manuscript here: http://www.dovepress.com/international-journal-of-chronic-obstructive-pulmonary-disease-journal 